American Medical Journal 3 (2): 115-123, 2012

ISSN 1949-0070

C 2012 Science Publications

\title{
What Is The "Safest" Non-Steroidal Anti-Inflammatory Drugs?
}

\author{
${ }^{1}$ Patompong Ungprasert, \\ ${ }^{1}$ Wonngarm Kittanamongkolchai, ${ }^{1}$ Chrystal Price, ${ }^{1}$ Supawat Ratanapo, \\ ${ }^{1}$ Napat Leeaphorn, ${ }^{1}$ Daych Chongnarungsin and ${ }^{1}$ Wisit Cheungpasitporn, \\ ${ }^{1}$ Department of Internal Medicine, \\ Bassett Medical Center and Columbia University, \\ College of Physicians and Surgeons, Cooperstown, New York
}

\begin{abstract}
Non-Steroidal Anti-Inflammatory Drugs (NSAIDs) which are widely used in clinical practice are also very well-known for their various adverse reactions. Each NSAID has its own unique safety profile and selecting an appropriate NSAID must be individualized for each patient based on his or her medical needs and risk factors. We reviewed literatures on NSAIDs, focusing on their adverse reaction profile. We reviewed and compared the incidence of adverse reaction from individual NSAIDs according to organ systems. This review includes both selective COX-2 inhibitors and nonselective NSAIDs. Based on the most up-to-date evidence, ibuprofen appears to be the preferred NSAIDs based on its favorable GI and nephrotoxicity profiles. Naproxen might be considered in patients who have greater cardiac risk. Celecoxib, at the dose of less than or equal to $200 \mathrm{mg} \mathrm{day}^{-1}$, might be an option in the patients who are at high risk for GI bleeding.
\end{abstract}

Key word: Non-Steroidal Anti-Inflammatory Drugs (NSAIDs), COX-2 inhibitor, adverse drug reaction, adverse reactions, safety profile, clinical practice, organ systems

\section{INTRODUCTION}

Non-Steroidal Anti-Inflammatory Drugs (NSAIDs) are the most commonly used medications in the United States (Laine, 2001). They are primarily used as pain killer as well as anti-inflammatory agent. Currently, there is nineteen NSAIDs available in the United States (Table 1). With the continued aging of our population, the Center for Disease Control predicts that the prevalence of painful degenerative joint disease will increase, which will probably lead to an increase in the use of NSAIDs.

NSAIDs are well-known for their adverse drug reaction. Approximately $30 \%$ of hospitalizations of adverse drug reactions are caused by NSAIDs (Pirmohamed et al., 2004). Adverse reactions can affect various organ systems with gastrointestinal bleeding and cardiovascular events being the most serious and sometimes fatal reaction.

Most of the toxic effects of NSAIDs are a direct result of their mode of action which includes suppression of prostaglandins synthesis. Cyclooxygenase (COX) is the key enzyme in prostaglandin synthetic process that is inhibited by NSAIDs.
Table 1: Available NSAIDs in the United States and their usual dose

\begin{tabular}{|c|c|}
\hline Generic name & Usual dose \\
\hline \multicolumn{2}{|c|}{ Selective COX-2 inhibitor } \\
\hline Celecoxib & $100-200 \mathrm{mg} \mathrm{day}{ }^{-1}$ \\
\hline \multicolumn{2}{|c|}{ Non selective COX-2 inhibitor } \\
\hline Aspirin & 2.6-6 g day 4-5 divided doses \\
\hline Diclofenac & $50 \mathrm{mg}$ BID \\
\hline Diflunisal & $0.25-0.75 \mathrm{~g} \mathrm{BID}$ \\
\hline Etodolac & 200-300 mg BID-QID \\
\hline Fenoprofen & 300-600 mg QID \\
\hline Flurbiprofen & $100 \mathrm{mg}$ BID-TID \\
\hline Ibuprofen & $200-800 \mathrm{mg}$ QID \\
\hline Indomethacin & 25-50 mg TID-QID \\
\hline Ketoprofen & 75 mg TID \\
\hline Meclofenamate & 50-100 mg TID-QID \\
\hline Mefenamic acid & $250 \mathrm{mg}$ QID \\
\hline Meloxicam & $7.5-15 \mathrm{mg}$ OD \\
\hline Nabumetone & 500 mg BID \\
\hline Naproxen & $250-500 \mathrm{mg}$ BID \\
\hline Oxaprozin & $600 \mathrm{mg}$ OD \\
\hline Piroxicam & $10-20 \mathrm{mg}$ OD \\
\hline Sulindac & $150-200 \mathrm{mg}$ BID \\
\hline Tolmetin & 400-600 mg TID \\
\hline Ketorolac & $\begin{array}{l}\text { IV or IM } 30 \mathrm{mg} \mathrm{q}^{6} \mathrm{hr} \\
\left(\max 120 \mathrm{mg} \mathrm{day}^{-1}\right)\end{array}$ \\
\hline
\end{tabular}

COX enzyme has two isoforms COX-1 and COX-2. COX-1 is expressed in most tissues; it serves as a housekeeping enzyme responsible for normal cell homeostasis. COX-2 is expressed in a limited number 
of organs in a normal physiologic condition but its expression is inducible with inflammation. The discovery of the two isoforms of the COX enzyme lead to the development of selective anti COX-2 NSAIDs with the hope of a reduction in adverse effects, most specifically gastrointestinal bleeding (Flower, 2003).

Naming the universal "safest" NSAID deems difficult because each NSAID has its own different adverse reaction profile; "safety" priorities should be individualized based upon patient's concurrent illnesses. This review will discuss the potential adverse reactions of both conventions (non-selective) and selective NSAIDs according to specific organ systems.

Cardiovascular effect: During the last 10 years, the adverse cardiovascular effects of selective COX-2 inhibitors have been a highly debated issue. In October 2004, rofecoxib was withdrawn from US and world market after a randomized placebo-controlled trial found that it increased the incidence of Myocardial Infarction (MI) and sudden cardiac death (Bresalier et al., 2005). Attention has now turned to the cardiovascular safety profile of the remaining selective COX-2 inhibitor (celecoxib) and non-selective NSAIDs. McGettigan and Henry (2006) conducted a meta-analysis of an observational study and found that celecoxib at doses of less than or equal to $200 \mathrm{mg} \mathrm{day}^{-1}$ appeared to be safe and did a not significantly increase the risk of MI and sudden cardiac death. Conventional NSAIDs including ibuprofen, indomethacin and piroxicam, also did not increase the cardiac risk. Of significant concern from this meta-analysis is, diclofenac statistically increased the risk of cardiovascular events with a Relative Risk (RR) of 1.36 (95\% CI 1.21-1.54). A possible explanation for the disparate result is that diclofenac is the most selective for COX-2 compared with other conventional NSAIDs. This study also showed a slight cardioprotective effect of naproxen but without statistical significance (Table 2). Another meta-analysis which included only randomized data revealed the same increased cardiac risk from diclofenac and revealed no significant cardioprotective benefit from naproxen. However, this study found a significant increased risk with a daily dose of celecoxib more than or equal $400 \mathrm{mg}$ (Kearnry et al., 2006).

Use of NSAIDs can worsen pre-existing heart disease. The risk of worsening heart failure is well illustrated in an observational study which found that all the NSAIDs in the study (rofecoxib, celecoxib, ibuprofen, diclofenac and naproxen) significantly increased the incidence of death and re-hospitalization because of heart failure and/or MI (Gislason et al., 2009). The mechanism by which NSAIDs can cause exacerbation of heart failure is related to their vasoconstrictive effect leading to systemic hypertension resulting in increased afterload (Dzau et al., 1984).

Furthermore, non-aspirin NSAIDs can interfere with the beneficial anti-platelet effect of aspirin. Concomitant administration of reversible COX-1 inhibitors may prevent irreversible platelet inhibition by low-dose aspirin, due to competition between theses drug and aspirin for a common binding site on COX-1. At least two of conventional NSAIDs (ibuprofen and naproxen) have been found to have this drug interaction in vitro. This pharmacodynamic interaction is not seen with selective COX-2 inhibitor or with diclofenac, which has some degree of COX-2 selectivity (CatellaLawson et al., 2001; Capone et al., 2005). Whether this interaction attenuates the cardioprotective benefit of low-dose aspirin is unclear (Corman et al., 2005; Rodiguez et al., 2004).

In conclusion, based on available data from the mentioned study, NSAIDs should be avoided in patients with MI or heart failure. In patients with lower cardiac risk, NSAIDs might be used with caution with the lowest effective dose and shortest possible duration. Diclofenac should be avoided given the clear evidence of increase incidence of MI and sudden cardiac death. The only available selective COX-2 inhibitor, Celecoxib, appears to be safe with the daily dose of not more than $200 \mathrm{mg} \mathrm{day}^{-1}$. Naproxen might be considered for use in this group of patients given its slight cardioprotective benefit, though this consideration is based on data which lack statistical significance.

Gastrointestinal effects: Aspirin and other non-selective NSAIDs are well-known for their gastrointestinal toxicities ranging from asymptomatic mucosal injury to fatal upper GI bleeding. The toxicity comes from the mode of action of COX-1 inhibition. COX-1 is constitutively expressed in gastric and duodenal mucosa and is responsible for mucoprotective prostaglandins synthesis. As a result of COX-1 inhibition and subsequent reduction of prostaglandins synthesis, gastric and duodenal mucosa which appears to be more vulnerable to luminal acid and pepsin (Cryer and Feldman, 1998; Jick, 1981). These gastrointestinal toxicities result in more than 100,000 hospital admissions and 7,000-10,000 deaths per year in the United States.

Aspirin, which is now widely used for coronary artery disease treatment and prevention, can cause significant gastric mucosa damage even at very low doses. One study in healthy human subjects found that aspirin at a dose of $10 \mathrm{mg}$ day $^{-1}$ reduced gastric mucosal prostaglandins to $40 \%$ of baseline level and induced significant gastric mucosa injury (Cryer and Feldman, 1999). Several epidemiological and placebocontrolled studies showed the dose-response relationship between clinically significant gastric events and the dose of aspirin (Jick, 1981; Farrell et al., 1991; Weil et al., 1995; Singh and Triadafilopoulos 1999). 
Am. Med. J. 3 (2): 115-123, 2012

Table 2: Relative risk and 95\% confidence interval on cardiovascular events for each NSAID (adapted from Mcgettigan et al., 2006)

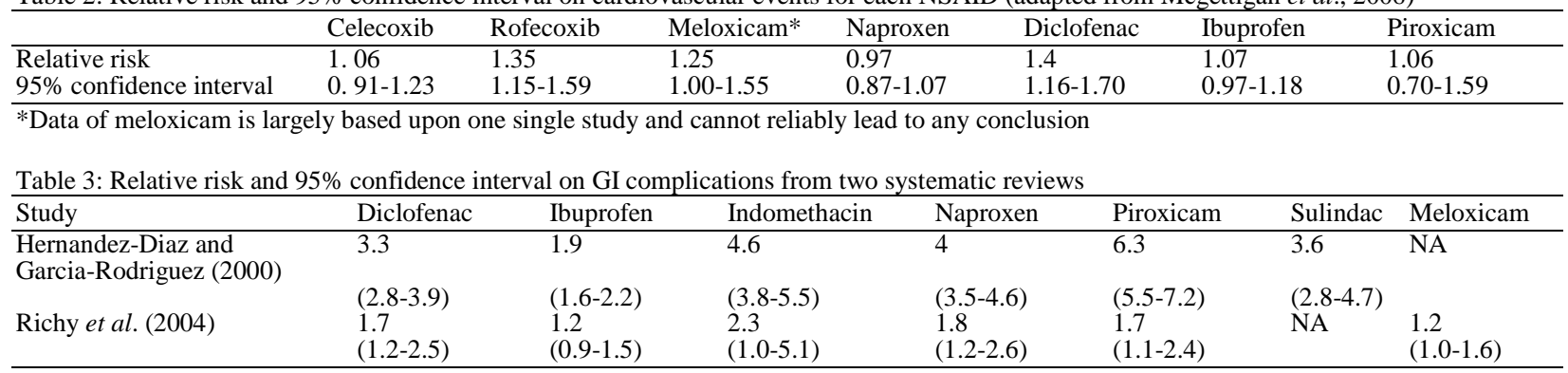

Table 4: NSAIDs GI toxicity risk stratification (adapted from Lanza et al., 2009)

High risk

\begin{tabular}{ll}
\hline & $\begin{array}{l}\text { History of previous complicated ulcer, } \\
\text { especially recent } \\
\text { Multiple }(>2) \text { risk factors }\end{array}$ \\
& Age $>65$ years \\
(1-2 risk factors) & $\begin{array}{l}\text { High dose NSAID therapy } \\
\text { A previous history of uncomplicated ulcer } \\
\text { Concurrent use of aspirin (including low-dose), } \\
\text { corticosteroid or anticoagulant } \\
\text { Low risk }\end{array}$ \\
\hline
\end{tabular}

In contrast to irreversible COX inhibition of aspirin, most NSAIDs inhibit COX-1 and COX-2 reversibly. Nevertheless, transient COX-1 inhibition is sufficient to make gastric mucosa vulnerable to injury (Silverstein $e t$ al., 2000). The risks of upper GI toxicity associated with non-selective NSAIDs have been extensively studied. A meta-analysis of observational studies has shown that the risk of upper GI complications is increased 4-fold among NSAIDs users, with the highest risk during the first month of treatment (Hernandez-Diaz and GarciaRodriguez, 2000). Risk factors for NSAIDs-related bleeding include age > 60 years, high-dose NSAID treatment, a previous history of peptic ulcer, concurrent use of low-dose aspirin, anti-coagulants or steroids and H. pylori infection (Hernandez-Diaz and GarciaRodriguez, 2000; Huang et al., 2002)

From the same meta-analysis, ibuprofen was associated with the lowest risk, followed by diclofenac, sulindac, naproxen, indomethacin and ketoprofen, while piroxicam conferred the highest risk (Hernandez-Diaz and Garcia-Rodriguez, 2000). Another meta-analysis of controlled trials revealed similar results. This study found that ibuprofen and meloxicam were the only commonly used non-selective NSAIDs that did not significantly increase the risk of bleeding while indomethacin provided the highest risk for GI complication, followed by naproxen, diclofenac and piroxicam as illustrated in Table 3 (Richy et al., 2004).

Selective COX-2 inhibitor has been developed to overcome the gastrointestinal toxicities of non-selective
NSAIDs. Several studies have shown favorable safety profile of this selective NSAID with respect to upper GI bleeding. A systematic review of randomized controlled trials has shown that COX-2 selective inhibitors produced significantly fewer gastroduodenal ulcers and clinically important ulcer complications compared with non-selective NSAIDs (Rostom et al., 2007). However, the co-administration of low-dose aspirin significantly reduced this safety advantage (Rostom et al., 2007; Singh et al., 2006). However, concerns raised regarding cardiovascular adverse effects of selective COX-2 inhibitor have limited their clinical use.

A number of clinical trials have studied a variety of options to prevent gastroduodenal ulcer and bleeding in the patients indicated for NSAIDs. Two meta-analyses of placebo-controlled studies found that the risk of symptomatic ulcer was significantly reduced by Proton Pump Inhibitors (PPI), misoprostol and COX-2 inhibitors (Rostom et al., 2002; Koch et al., 1996). The evidence for the effectiveness of Histamine-2 Receptor Antagonists (H2RA) is still unclear. The same metaanalysis revealed no gastric ulcer prevention benefit of a standard dose of H2RA (Koch et al., 1996) but some studies showed a decrease in gastric ulcer incidence with double-dose H2RA. However, these studies were shortterm (12-24 weeks) and focused on endoscopic finding rather than clinical endpoints (Taha et al., 1996; 2009).

In 2009, the American College of Gastroenterology issued an official guideline for prevention of NSAIDrelated ulcer complications. This guideline emphasizes on balancing between patient's cardiovascular risk and GI toxicity risk. Patients are stratified into low, moderate and high GI toxicity risk based upon the number of risk factors they have as illustrated in Table 4. Cardiovascular risk is arbitrarily categorized into low and high risk based upon the patients' need to use lowdose aspirin to prevent serious cardiovascular events. These recommendations are summarized in Table 5.

In general, patient with low cardiac risk and without GI risk factors can use non-selective NSAID alone without protective measures. The least ulcerogenic NSAIDs like ibuprofen and meloxicam are 
preferable. In the patient without GI risk factors but is at high risk for cardiovascular events, naproxen is preferred based upon their more favorable cardiac safety profile. In this group of patient, protective measure either PPI or misoprostal should be employed because naproxen confers more considerable GI toxicity compared with ibuprofen.

A patient who is classified as moderate risk should have mucoprotective agent, either PPI or mesoprostal, while using conventional NSAIDs. If a patient has low cardiac risk, COX-2 inhibitor alone can be used as an alternative. Again, naproxen is the preferable if the patient has high cardiac risk. For the patient who is at high risk for both GI and cardiac event, NSAIDs should be entirely avoided. If the patient has only high GI risk but has low cardiac risk, COX-2 inhibitor plus PPI or mesoprostal can be used with caution (Lanza et al., 2009).

In addition, Helicobacter pylori infection is another independent risk factor. Several studies suggest that $\mathrm{H}$. pylori infection increases the risk of peptic ulcer disease in patients taking NSAIDs (Chan, 2001; Bazzoli et al., 2001 ; Huang et al., 2002) and eradication of H. pylori decreased the incidence of NSAID-related ulcer (Hawkey et al., 1998; Chan et al., 2001). Based on these evidences, it is advisable that all the patients who require long-term NSAIDs should be tested and treated for H. Pylori.

Hepatotoxicity: Hepatic adverse reaction is relatively uncommon. The incidence of serious reaction requiring hospitalization ranges from 3-23 cases per 100,000 person-year (Rodriguez et al., 1992; Traversa et al., 2003). Hepatotoxicity from NSAIDs (except for aspirin) appears to be idiosyncratic and can present with either hepatocellular or cholestatic pattern (Rubenstein and Laine, 2004). Nimesulide, which has never been approved by FDA and not available in the United States, is particularly associated with increased risk of hepatic injury. A recent large cohort study showed that only nimesulide significantly increased the risk of hepatic injury while other NSAIDs in the study, including diclofenac, piroxicam, ketoprofen, ketorolac, ibuprofen, naproxen, celecoxib, meloxicam, cinnoxicam and flurbiprofen, tended to increase the risk but without statistical significance (Traversa et al., 2003). Another large case-control trial which studied on diclofenac, piroxicam, ibuprofen, naproxen, celecoxib and meloxicam showed that only sulindac significantly increased the incidence of hepatitis required hospitalization while the rest tended to increase the incidence but, again, without statistical significance (Gutthann and Rodigruez, 1993).

In the light of evidence of risk of hepatotoxicity and availability of alternative NSAIDs, nimesulide and sulindac should not be prescribed especially in patient with known liver disease such as chronic viral hepatitis, concurrent use of other hepatotoxic medications including alcohol and cirrhosis.

Nephrotoxicity: One of the most recognized adverse effects of NSAIDs is nephrotoxicity. Inhibition of COX can lead to renal impairment and dysregulation of water and electrolyte homeostasis. COX regulates renal hemodynamics, Glomerular Filtration Rate (GFR) and mediates salt and water excretion. Prostaglandins, generated through the action of COX, dilate the renal vascular bed, lower renal vascular resistance and increase renal perfusion (Dunn et al., 1998). However, they play only a minor role in maintaining renal blood flow in healthy persons. These prostaglandins will be critically important in maintaining the GFR in the situation of decreased effective circulatory volume. Thus, inhibiting prostaglandins by NSAIDs can contribute to a further decrease in overall renal perfusion causing renal impairment (Patrono and Dunn, 1987). Nevertheless, these renal effects are usually completely reversible following prompt discontinuation of the offending NSAIDs.

COX-2 inhibitors appear to have similar renal effects as nonselective NSAIDs and do not offer any renal safety benefits over nonselective NSAIDs. A recent large nested case-control study demonstrated a comparable relative risk of acute renal failure among rofecoxib user $(R R=2.31)$, naproxen user $(R R=2.42)$ and other nonselective NSAIDs user $(\mathrm{RR}=2.30)$ though the relative risk of celecoxib was marginally lower $(\mathrm{RR}=1.54)$ (Schneider et al., 2006).

Although there is no large epidemiological study to compare the relative risk of NSAIDs, low dose overthe-counter ibuprofen appears to be safe in most healthy subjects (Mann et al., 1993). Sulindac has its unique metabolic pathway that may spare renal oxygenase by oxidizing sulindac sulfide to an inactive metabolite in the kidney. Nonetheless, this capacity varies between individuals, which may explain conflicting reports on the effects of sulindac on urinary prostaglandins and renal function (Brandli et al., 1991).

Acute Interstitial Nephritis (AIN) is another common renal adverse effect. The mechanism by which NSAIDs can induce AIN is not well understood. One explanation is that inhibition of COX causes preferential conversion of arachidonic acid to leukotrienes resulting in helper T-cell activation and inflammation. Affected patients typically present with markedly elevated serum creatinine and proteinuria that occur several months after the introduction of NSAIDs. Renal function usually improves within 1-3 months after discontinuing the drug (Brezin et al., 1979). 
Am. Med.J. 3 (2): 115-123, 2012

Table 5: Summary of recommendations for prevention of NSAIDs-related ulcer complications (adapted from Lanza et al., 2009)

\begin{tabular}{|c|c|c|c|}
\hline & \multicolumn{3}{|l|}{ GI risk } \\
\hline & Low & Moderate & High \\
\hline Low $\mathrm{CV}$ risk & $\begin{array}{l}\text { NSAID alone (the least ulcerogenic NSAID } \\
\text { at the lowest effective dose) }\end{array}$ & $\begin{array}{l}\text { SAID + PPI or } \\
\text { misoprostal }\end{array}$ & $\begin{array}{l}\text { Alternative therapy if possible or } \\
\text { COX-2 inhibitor + PPI or misoprostal }\end{array}$ \\
\hline High CV risk & Naproxen + PPI or misoprostal & $\begin{array}{l}\text { Naproxen + PPI or } \\
\text { Misoprostal }\end{array}$ & $\begin{array}{l}\text { Avoid NSAIDs and COX-2 inhibitors. } \\
\text { use alternative therapy }\end{array}$ \\
\hline
\end{tabular}

Table 6: Relative risk and 95\% confidence interval on cerebrovascular events from observational studies (adapted from Roumie et al., 2008)

\begin{tabular}{|c|c|c|c|c|c|c|c|}
\hline Study & N stokes & Celecoxib & Rofecoxib & Naproxen & Ibuprofen & Diclofenac & Indomethacin \\
\hline Bak et al. (2003) Hemorrhagic & 867 & NA & NA & $0.8(0.3-2.1)$ & $1.3(0.9-2.0)$ & $1.3(0.6-2.8)$ & $1.3(0.5-3.7)$ \\
\hline Bak et al. (2003) Ischemic & 2171 & NA & NA & $0.7(0.4-1.1)$ & $1.3(1.0-1.6)$ & $1.1(0.7-1.7)$ & $1.4(0.8-2.4)$ \\
\hline Andersohn et al. (2006) & 3094 & $1.07(0.79-1.44)$ & $1.71(1.33-2.18)$ & $1.16(0.80-1.70)$ & $1.12(0.91-1.37)$ & $1.32(1.10-1.57)$ & NA \\
\hline Solomon et al. (2006) & 3552 & $1.00(0.92-1.09$ & $1.15(1.04-1.26)$ & $0.83(0.67-1.04)$ & $0.95(0.78-1.16)$ & $0.98(0.75-1.29)$ & NA \\
\hline Lee et al. (2007) & 4787 & $0.97(0.68-1.37)$ & $1.45(1.10-1.92)$ & $1.15(1.01-1.31)$ & $1.11(0.99-1.25)$ & $1.24(0.95-1.63)$ & $1.13(0.86-1.50)$ \\
\hline Roumie et al. (2008) & 4354 & $1.12(0.83-1.52)$ & $1.46(1.08-1.98)$ & $1.02(0.73-1.42)$ & $1.26(0.87-1.81)$ & $0.31(0.04-2.18)$ & $1.29(0.53-3.09)$ \\
\hline
\end{tabular}

Virtually all NSAIDs can cause AIN but fenoprofen appears to be associated with a higher risk. It is responsible for $47 \%$ of AIN caused by NSAIDs in a case series (Porile et al., 1990).

Nephrotic syndrome is also well-known to be associated with the use of NSAIDs. Renal pathology typically reveals minimal change disease though membranous nephropathy has been reported (Warren et al., 1989; Radford et al., 1996). Papillary necrosis, though rare nowadays, is the only permanent complication of NSAIDs and usually occurs after long-term use.

NSAIDs can interfere with normal electrolyte and water homeostasis but usually without significant clinical outcomes. NSAIDs reduces sodium excretion and blunt the diuretic effect of loop diuretics, but these effects are usually mild and subclinical (Clive and Stoff, 1984). NSAIDs attenuate the release of renin and hence reduce aldosterone production, results in decrease potassium excretion. The risks of hyperkalemia are further potentiated by renal dysfunction, diabetes, congestive heart failure and the use of potassium supplement, ACE inhibitors and/or potassium sparing diuretics (Whelton and Hamilton, 1991). Last, long term use of NSAIDs can slightly increase mean arterial pressure by $5 \mathrm{mmHg}$ and interfere with several antihypertensive medications such as diuretics, beta-blockers and ACE inhibitors (Johnson et al., 1994).

Central nervous system: The same study that has led to withdrawal of rofecoxib from worldwide market also revealed an increased incidence of stroke among rofecoxib users though without statistical significance (Bresalier et al., 2005). Subsequent observational studies showed a significant increase of cerebrovascular risk among rofecoxib user and did not show any significant increase of the risk among celecoxib user (Andersohn et al., 2006; Solomon et al. 2006; Lee et al., 2007; Roumie et al., 2008). The results regarding conventional non-aspirin NSAIDs were less consistent. Though the majority of studies did not show any increased risk for either ischemic or hemorrhagic stroke with the use of any conventional non-aspirin NSAIDs (Bak et al., 2003; Solomon et al., 2006; Roumie et al., 2008) Andersohn et al. (2006) reported an increased risk for ischemic stroke with the use of diclofenac and Lee et al. (2007) reported an increased risk for cerebrovascular events among users of naproxen. Result from these studies are summarized in Table 6.

In addition to cerebrovascular events, NSAIDs can cause other CNS adverse effects. Aseptic meningitis is the infrequent adverse effect of NSAID. It is found most commonly in patients with lupus treated with ibuprofen, but it should be considered in any patient with meningitis if the patient has used NSAIDs. Psychosis and cognitive impairment in association with NSAIDs use have been reported, particularly in elderly patient started on a regimen of indomethacin. Thus, NSAIDs (especially indomethacin) should be prescribed with caution in this population with close attention to mental status changes (Hoppmann et al., 1991).

Hematologic effect: The most significant hematologic effect of NSAID use is the anti-platelet by inhibition of COX-1 which is responsible for Thromboxane A2 (TXA2) production. TXA2 is synthesized and released by platelets in response to a variety of stimuli and provides a mechanism for amplifying the platelet response leading to platelet aggregation (Hamburg et al., 1975). This anti-platelet effect of TXA2 inhibition provides therapeutic applications for thrombosis prevention in patients with coronary artery disease or peripheral vascular disease. Selective COX-2 inhibitors have minimal to no effect on platelet function since matured platelet expresses only COX-1. 
In the patient with thrombocytopenia (platelet count $<50,000 / \mathrm{microL})$ or platelet dysfunction, nonselective NSAIDs should be avoided; COX-2 inhibitors are considered as a safer option. Conventional NSAIDs should be discontinued four to five times their half-life before surgery to avoid an increased intra-operative and post-operative bleeding risk. Aspirin should be discontinued at least one week before surgery to allow the bone marrow to produce new platelets.

Neutropenia, although uncommon, is another adverse hematologic effect from NSAID use. A casecontrol study showed a significant increase in incidence of neutropenia among NSAID users, although no single class of NSAID or any individual NSAIDs was associated with a unique risk.

Cutaneous reaction: NSAIDs can cause several kinds of drug eruptions ranging from a mild pruritic rash to more severe skin reactions such as Toxic Epidermal Necrolysis (TEN) and Steven Johnson Syndrome (SJS). The true incidence of skin reactions related to NSAIDs is difficult to estimate, as these medications are commonly purchased over-the-counter, with minor skin side effects going quite often underreported. However, in one prospective study of nearly 20,000 inpatients who were prescribed NSAIDs, $0.3 \%$ of the participants were found to have a NSAIDs-related skin reaction. Maculopapular rash was the most common skin reaction, followed by urticaria, angioedema, serum sickness syndromes and erythema nodosum (Kaiser et al., 1987).

Hypersensitivity reaction is a unique skin condition caused by NSAIDs. Symptoms include facial angioedema, urticaria, conjunctivitis as well as respiratory symptoms such as rhinorrhea, cough and bronchospasm. Again, one explanation is that inhibition of COX-1 leads to a shunting of arachidonic acid metabolism, causing overproduction of leukotrienes consequently resulting in allergy-like effects (Keane et al., 1984). However, the reasons why only a certain number of people react to NSAIDs are not completely clear. The known predisposing factors putting certain people at risk for skin reactions include an atopic diathesis, female sex, young adulthood, chronic urticaria and the use of the NSAIDS for the relief of acute pain (Sanchez-Borges et al., 2002). Drugs that inhibit COX-2 with higher specificity than classic NSAIDs are more tolerated by the majority of classic NSAID-sensitive patients. Nevertheless, hypersensitivity reactions have been reported with COX-2 inhibitors as well (Dona et al., 2011).

Pseudoporphyria is a photosensitive reaction of skin with tense bullae and erosions which is clinically indistinguishable from true cutaneous porphyria tarda. However, there is no abnormality detected in porphyrin metabolism with pseudoporphyria. It has been found to be associated with several NSAIDs, including naproxen, nabumetone and ketoprofen (Green and Manders, 2001). This reaction is believed to be caused by the photosensitizing drug that behaves in a similar fashion to photoactivated endogenous porphyrins and target similar structures in the skin (Zelickson, 1964).

With regards to severe skin reactions, the risk of SJS and TEN caused by NSAIDs is extremely low, which is less than 1-2 per 1 million users per week. Oxicam derivatives appear to have the greatest association with SJS and TEN (Ward et al., 2010). There is also a strong association between SJS/TEN and the use of the sulfonamide COX-2 inhibitors, particularly valdecoxib (Grenade et al., 2005). Aspirin is not associated with a measurable increase in the risk of these rare but severe reactions (Kaufman and Kelly, 2001).

As with most drug-induced skin reactions, the best approach to the management of NSAD-induced skin reactions is to withdraw the trigger medication. This sometimes results in resolution of the rash, although this may take some months and is often not universal.

Pulmonary effect: Non-aspirin NSAIDs rarely causes adverse pulmonary effects, though they can precipitate acute exacerbations of airway inflammation in patients with Aspirin Exacerbated Respiratory Disease (AERD). AERD is characterized by persistent and severe inflammation of the upper and lower respiratory tracts manifested as asthma and chronic rhinosinusitis with nasal polyposis inducible by aspirin or NSAIDs ingestion. This reaction is directly related to $\mathrm{COX}-1$ inhibition; selective COX-2 inhibitors are much less likely to trigger AERD (Palikhe et al., 2009).

\section{CONCLUSION}

Selecting the most appropriate NSAIDs for each patient should be tailored to each individual patient based on the patient's medical background. In general, ibuprofen is the preferred NSAIDs based on its favorable GI and nephrotoxicity profiles. Naproxen might be considered in patients who have greater cardiac risk. Celecoxib, at the dose of less than or equal to $200 \mathrm{mg}$ day $^{-1}$, might be an option in the patients who are at high risk for GI bleeding. However, both selective and nonselective NSAIDs should be avoided in patients who have a MI, heart failure and chronic kidney disease.

\section{REFERENCES}

Laine, L., 2001. Approaches to nonsteroidal antiinflammatory drug use in the high-risk patient. Gastroenterology, 120: 594-606. PMID: 11179238 
Pirmohamed, M., S. James, S. Meakin, C. Green and A.K. Scott et al., 2004. Adverse drug reactions as cause of admission to hospital: Prospective analysis of 18820 patients. BMJ, 329:15-19. PMID: 15231615

Flower, R.J., 2003. The development of COX2 inhibitors. Nat. Rev. Drug Discov., 2: 179-191. PMID: 12612644

Bresalier, R.S., R.S. Sandler, H. Quan, J.A. Bolognese and B. Oxenius et al., 2005. Cardiovascular events associated with rofecoxib in a colorectal adenoma chemoprevention trial. N Eng. J. Med., 352: 10921102. PMID: 15713943

McGettigan, P. and D. Henry, 2006. Cardiovascular risk and inhibition of cyclooxygenase: a systematic review of the observational studies of selective and nonselective inhibitors of cyclooxygenase 2 . JAMA, 296: 1633-1644. PMID: 16968831

Kearnry, P.M., C. Baigent, J. Goldwin, H. Hall and J.R. Emberson et al., 2006. Do selective cyclooxygenase-2 inhibitors and traditional nonsteroidal anti-inflammatory drugs increase the risk of atherothrombosis? Meta-analysis of randomised trials. BMJ, 332: 1302-1308. PMID: 16740558

Gislason, G.H., J.N. Rasmussen, S.Z. Abildstrom, T.K. Schramm and M.L. Hansen et al., 2009. Increased mortality and cardiovascular morbidity associated with use of nonsteroidal anti-inflammatory drugs in chronic heart failure. Arch. Int. Med., 169: 141149. PMID: 19171810

Dzau, V.J., M. Packer, L.S. Lilly, S.L. Swartz and N.K. Hollenberg et al., 1984. Prostaglandins in severe congestive heart failure. relation to activation of the renin--angiotensin system and hyponatremia. $\mathrm{N}$ Engl. J. Med., 310: 347-352. PMID: 6361570

Catella-Lawson, F., M.P. Reilly, S.C. Kapoor, A.J. Cucchiara and S. DeMarco et al., 2001. Cyclooxygenase inhibitors and the antiplatelet effects of aspirin. N Engl. J. Med., 345: 1809-1817. PMID: 11752357

Capone, M.L., M.G. Sciulli, S. Tacconelli, M. Grana and E. Riciotti et al., 2005. Pharmacodynamic interaction of naproxen with low-dose aspirin in healthy subjects. J. Am. Coll. Cardiol., 45: 12951301. PMID: 15837265

Corman, S.L., B.A. Fedutes and N.T. Ansani, 2005. Impact of nonsteroidal antiinflammatory drugs on the cardioprotective effects of aspirin. Ann. Pharmacother., 39: 1073-1079. PMID: 15870140

Rodiguez, L.A.G., C. Varas-Lorenzo, A. Maguire and A. Gonzalez-Perez, 2004. Nonsteroidal antiinflammatory drugs and the risk of myocardial infarction in the general population. Circulation, 109: 3000-3006. PMID: 15197149
Cryer, B. and M. Feldman, 1998. Cyclooxygenase-1 and cyclooxygenase-2 selectivity of widely used nonsteroidal anti-inflammatory drugs. Am. J. Med., 104: 413-421. PMID: 9626023

Jick, H., 1981. Effects of aspirin and acetaminophen in gastrointestinal hemorrhage. Results from the Boston Collaborative Drug Surveillance Program. Arch Int. Med., 141: 316-321. PMID: 6970559

Cryer, B. and M. Feldman, 1999. Effects of very low dose daily, long-term aspirin therapy on gastric, duodenal and rectal prostaglandin levels and on mucosal injury in healthy humans. Gastroenterology, 117: 17-25. PMID: 10381905

Farrell, B., J. Godwin, S. Richards and C. Warlow, 1991. The United Kingdom transient ischemic attack or minor ischemic attack (UK-TIA) aspirin trial: Final results. J. Neurol Neurosurg Psychiatry, 54: 1044-1054. PMID: 1783914

Weil, J., D. Colin-Jones, M. Langman, D. Lawson and R. Logan, et al., 1995. Prophylactic aspirin and risk of peptic ulcer bleeding. BMJ, 310: 827-830. PMID: 7711618

Singh, G. and G. Triadafilopoulos, 1999. Epidemiology of NSAID induced gastrointestinal complications. J. Rheumatol Suppl., 56: 18-24. PMID: 10225536

Silverstein, F.E., G. Faich, J.L. Goldstein, L.S. Simon and T. Pincus et al., 2000. Gastrointestinal toxicity with celecoxib vs nonsteroidal anti-inflammatory drugs for osteoarthritis and rheumatoid arthritis: the CLASS study: A randomized controlled trial. celecoxib long-term arthritis safety study. JAMA, 284: 1247-1255. PMID: 10979111

Hernandez-Diaz, S. and L.A. Garcia-Rodriguez, 2000. Association between nonsteroidal antiinflammatory drugs and upper gastrointestinal tract bleeding/perforation: An overview of epidemiologic studies published in the 1990s. Arch. Int. Med., 160: 2093-2099. PMID: 10904451

Huang, J.Q., S. Sridhar and R.H. Hunt, 2002. Role of Helicobacter pylori infection and non-steroidal anti-inflammatory drugs in peptic-ulcer disease: A meta-analysis. Lancet, 359: 14-22. PMID: 11809181

Richy, F., O. Bruyere, O. Ethgen, V. Rabenda and G. Bouvenot et al., 2004. The time dependent risk of gastrointestinal complications induced by nonsteroidal anti-inflammatory drug use: A consensus statement using a meta-analytic approach. Ann. Rheum Dis., 63: 759-766. PMID: 15194568

Rostom, A., K. Muir, C. Dube, E. Jolicoeur and M. Boucher et al., 2007. Gastrointestinal safety of cyclooxygenase-2 inhibitors: A Cochrane Collaboration systematic review. Clin. Gastroenterol Hepatol, 5: 818-828. PMID 17556027 
Singh, G., J.G. Fort, J.L. Goldstein, R.A. Levy and P.S. Hanrahan, et al., 2006. Celecoxib versus naproxen and diclofenac in osteoarthritis patients: SUCCESS-1 study. Am J. Med., 119: 255-266. PMID: 16490472

Rostom, A., C. Dube, G. Wells, P. Tugwell and V. Welch et al., 2002. Prevention of NSAID-induced gastroduodenal ulcers. Cochrane Database Syst. Rev., 4: CD002296-CD002296. PMID: 12519573

Koch, M., A. Dezi, F. Ferrario and I. Capurso, 1996. Prevention of nonsteroidal anti-inflammatory druginduced gastrointestinal mucosal injury. A metaanalysis of randomized controlled clinical trials. Arch Int. Med., 156: 2321-2132. PMID: 8911239

Taha, A.S., N. Hudson, C.J. Hawkey, A.J. Swannell and P.N. Trye et al., 1996. Famotidine for the prevention of gastric and duodenal ulcers caused by nonsteroidal antiinflammatory drugs. N Engl. J. Med., 334: 1435-1439. PMID: 8618582

Taha, A.S., C. McCloskey, R. Prasad and V. Bezlyak, 2009. Famotidine for the prevention of peptic ulcers and oesophagitis in patients taking low-dose aspirin (FAMOUS): A phase III, randomized, double-blind, placebo-controlled trial. Lancet, 374 : 119-125. PMID: 19577798

Lanza, F.L., F.K. Chan and E.M. Quigley, 2009. Guidelines for prevention of NSAID-related ulcer complications. Am. J. Gastroenterol, 104: 728-738. PMID: 19240698

Chan, F.K., 2001. Helicobacter pylori and nonsteroidal anti-inflammatory drugs. Gastroenterol Clin North Am, 30: 937-952. PMID: 11764536

Bazzoli, F., L.D. Luca and D.Y. Graham., 2001. Helicobacter pylori infection and the use of NSAIDs. Best Pract Res Clin Gastroenterol, 15: 775-785. PMID: 11566040

Hawkey, C.J., Z. Tulassay, L. Szczepanski, C.J. van Rensburg and A. Filipowicz-Sosnowska et al., 1998. Randomized controlled trial of Helicobacter pylori eradication in patients on non-steroidal antiinflammatory drugs: HELP NSAIDs study. Helicobacter Eradication for Lesion Prevention. Lancet, 352: 1016 -1021. PMID: 9759744

Chan, F.K., S.C. Chung, B.Y. Suen, Y.T. Lee and W.K. Leung et al., 2001. Preventing recurrent upper gastrointestinal bleeding in patients with Helicobacter pylori infection who are taking lowdose aspirin or naproxen . N Engl. J Med, 344: 967-973. PMID: 11274623

Rodriguez, L.A.G., S. Perez Gutthann, A.M. Walker and L. Lueck, 1992. The role of non-steroidal antiinflammatory drugs in acute liver injury. BMJ, 305: 865-868. PMID: 1422399
Traversa, G., C. Bianchi, R.D. Cas, I. Abraha and F. Menniti-Ippolito et al., 2003. Cohort study of hepatotoxicity associated with nimesulide and other non-steroidal anti-inflammatory drugs. BMJ, 327: 18-22. PMID: 12842950

Rubenstein, J.H. and L. Laine, 2004. Systematic review: The hepatotoxicity of non-steroidal antiinflammatory drugs. Aliment Pharmacol Ther, 20: 373-80. PMID: 15298630

Gutthann, S.P. and L.A.G. Rodigruez, 1993. The increased risk of hospitalizations for acute liver injury in a population with exposure to multiple drugs. Epidemiology, 4: 496-501. PMID: 8268277

Dunn, M.J., M. Simonson, E.W. Davidson, L.A. Scharschmidt and J.R. Sedor, 1998. Nonsteroidal anti-inflammatory drugs and renal function. J. Clin Pharmacol, 28: 524-529.

Patrono, C. and M.J. Dunn, 1987. The clinical significance of inhibition of renal prostaglandin synthesis. Kidney Int, 32: 1-12. PMID: 3306093

Schneider, V., L.E. Lévesque, B. Zhang, T. Hutchinson and J.M. Brophy, 2006. Association of selective and conventional nonsteroidal antiinflammatory drugs with acute renal failure: A population-based, nested case-control analysis. Am J Epidemiol., 164: 881-889. PMID 17005625

Mann, J.F., M. Goerig, K. Brune and F.C. Luft, 1993. Ibuprofen as an over-the-counter drug: Is there a risk for renal injury? Clin Nephrol, 39: 1-6. PMID: 8428401

Brandli, D.W., E. Sarkissian, S.C. Ng and H.E. Paulus, 1991. Individual variability in concentrations of urinary sulindac sulfide. Clin Pharmacol Ther., 50: 650-655. PMID: 1752108

Brezin, J.H., S.M. Katz, A.B. Schwartz and J.L. Chinitz, 1979. Reversible renal failure and nephrotic syndrome associated with nonsteroidal anti-inflammatory drugs. N Engl. J. Med., 301: 1271-1273. PMID: 503131

Porile, J.L., G.L. Bakris and S. Garella, 1990. Acute interstitial nephritis with glomerulopathy due to nonsteroidal anti-inflammatory agents: a review of its clinical spectrum and effects of steroid therapy. J. Clin. Pharmacol., 30: 468-475. PMID: 2189904

Warren, G.V., S.M. Korbet, M.M. Schwartz and E.J. Lewis, 1989. Minimal change glomerulopathy associated with nonsteroidal antiinflammatory drugs. Am J. Kidney Dis., 13: 127-130. PMID: 2629709

Radford, M.G., Jr. K.E. Holley, J.P. Grande, T.S. Larson and R.D. Wagoner et al., 1996. Reversible membranous nephropathy associated with the use of nonsteroidal anti-inflammatory drugs. JAMA, 276: 466-469. PMID: 8691554 
Clive, D.M. and J.S. Stoff, 1984. Renal syndromes associated with nonsteroidal antiinflammatory drugs. N Engl J. Med., 310: 563-572. PMID: 6363936

Whelton, A. and C.W. Hamilton, 1991. Nonsteroidal anti-inflammatory drugs: Effects on kidney function. J. Clin Pharmacol., 31: 588-698. PMID: 1894754

Johnson, A.G., T.V. Nguyen and R.O. Day, 1994. Do nonsteroidal anti-inflammatory drugs affect blood pressure? A meta-analysis. Ann. Int. Med., 121: 289-300. PMID: 8037411

Andersohn, F., R. Schade, S. Suissa and E. Garbe, 2006. Cyclooxygenase-2 selective nonsteroidal anti-inflammatory drugs and the risk of ischemic stroke: A nested case-control study. Stroke, 37: 1725-1730. PMID: 16728684

Solomon, D.H., J. Avorn, T. Sturmer, R.J. Glynn and H. Mogun et al., 2006. Cardiovascular outcomes in new users of coxibs and nonsteroidal antiinflammatory drugs: High-risk subgroups and time course of risk. Arthritis Rheum, 54: 13781389. PMID: 16645966

Lee, T.A., B. Bartle and K.B. Weiss, 2007. The impact of nsaids on mortality and the effect of preexisting coronary artery disease in US veterans. Am J. Med., 120: e9-e16. PMID: 17208086

Roumie, C.L., E.F. Mitchel L. Jr, P.G. Kaltenbach and P. Arbogast, et al., 2008. Nonaspirin NSAIDs, cyclooxygenase 2 inhibitors and the risk for stroke. Stroke, 39: 2037-2045. PMID: 18436878

Bak, S., M. Andersen, I. Tsiropoulos, L.A. Garcia and J. Rodriguez, et al., 2003. Risk of stroke associated with nonsteroidal anti-inflammatory drugs: A nested case-control study. Stroke, 34: 379-386. PMID: 12574546

Hoppmann, R.A., J.G. Peden and S.K. Ober, 1991. Central nervous system side effects of nonsteroidal anti-inflammatory drugs. Aseptic meningitis, psychosis and cognitive dysfunction. Arch Int. Med., 151:1309-1313. PMID: 2064481

Hamburg, M., J. Svensson and B. Samuelsson, 1975. Thromboxanes: a new group of biologically active compounds derived from prostaglandin endoperoxides. Proc. Natl. Acad. Sci. USA., 72: 2994-2998. PMID: 1059088
Kaiser, U., J. Sollberger, R. Hoigné , R. Wymann and D. Fritschy, et al., 1987. Skin side effects of nonsteroidal anti-inflammatory analgesics and socalled minor analgesics. Report from the Berne Comprehensive Hospital Monitor. Schweiz Med. Wochenschr, 117: 1966-1970. PMID: 2962286

Keane, J.T., R.W. Pearson and F.D. Malkinson, 1984. Nalidixic acid-induced photosensitivity in mice: A model for pseudoporphyria. J. Invest. Dermatol., 82: 210-213. PMID: 6699423

Sanchez-Borges, M., A. Capriles-Hulett F. CaballeroFonseca, 2002. NSAID-induced urticaria and angioedema: A reappraisal of its clinical management. Am J. Clin Dermatol., 3: 599-607. PMID: 12444802

Dona, I., N. Blanca-López, L.R. Jagemann, M.J. Torres and C. Rondón et al., 2011. A response to a selective COX-2 inhibitor in patients with urticaria/angioedema induced by nonsteroidal antiinflammatory drugs. Allergy, 66: 1428-1433. PMID: 21834936

Green, J.J. and S.M. Manders, 2001. Pseudoporphyria. J. Am Acad Dermatol., 44: 100-108. PMID: 11148469

Zelickson, A.S., 1964. Phototoxic reaction with nalidixic acid. JAMA, 190: 556-557. PMID: 14198018

Ward, K.E., R. Archambault and T.L. Mersfelder, 2010. Severe adverse skin reactions to nonsteroidal antiinflammatory drugs: A review of the literature. Am J. Health Syst. Pharm, 67: 206-213. DOI: 10.2146/ajhp080603

Grenade, L.L., L. Lee, J. Weaver, R. Bonnel and C. Karwoski et al., 2005. Comparison of reporting of Stevens-Johnson syndrome and toxic epidermal necrolysis in association with selective COX-2 inhibitors. Drug Saf, 28: 917-924. PMID: 16180941

Kaufman, D.W. and J.P. Kelly, 2001. Acetylsalicylic acid and other salicylates in relation to StevensJohnson syndrome and toxic epidermal necrolysis. Br. J. Clin Pharmacol., 51: 174-176. PMID: 11259991

Palikhe, N.S., J.H. Kim and H.S. Park, 2009. Update on recent advances in the management of aspirin exacerbated respiratory disease. Yonsei Med. J., 50: 744-750. PMID: 20046412 